\title{
The Cardioband transcatheter annular reduction system
}

\author{
Matthew Miller, ${ }^{1,2}$, Vinod H. Thourani ${ }^{3}$, Brian Whisenant ${ }^{2}$ \\ ${ }^{1}$ University of Utah, Salt Lake City, UT, USA; ${ }^{2}$ Intermountain Heart Institute, Salt Lake City, UT, USA; ${ }^{3}$ MedStar Heart and Vascular Institute, \\ Georgetown University, Washington, DC, USA \\ Correspondence to: Brian Whisenant, MD. 5121 S Cottonwood Street, Salt Lake City, UT 84107, USA. Email: Brian.Whisenant@imail.org.
}

\begin{abstract}
Surgical implantation of a complete or incomplete ring to reduce the mitral valve annulus and improve leaflet coaptation is the foundation of valve repair for degenerative and functional mitral and tricuspid regurgitation. The Cardioband system (Edwards Lifesciences, Irvine, CA, USA) is a catheter-delivered annular reduction system that mimics the surgical approach. Sequential anchors are used to secure the Cardioband implant from trigone to trigone. The Cardioband implant is contracted under transesophageal guidance until valvular regurgitation is reduced. In the initial clinical experience, the Cardioband system was safely deployed in patients with clinically significant functional mitral regurgitation (MR) and yielded durable MR reduction, improved quality of life, and improved exercise capacity. The early and still-evolving Cardioband experience for tricuspid regurgitation is also promising. The ongoing pivotal ACTIVE Trial is designed to demonstrate the superiority of the Cardioband system to guideline-directed medical therapy in patients with clinically significant functional MR (FMR) using a composite endpoint of mortality, heart failure (HF) hospitalization, quality of life, and exercise capacity.
\end{abstract}

Keywords: Mitral regurgitation (MR); Cardioband; ACTIVE Trial; mitral annuloplasty; tricuspid regurgitation

Submitted Jul 17, 2018. Accepted for publication Oct 23, 2018.

doi: $10.21037 /$ acs.2018.10.10

View this article at: http://dx.doi.org/10.21037/acs.2018.10.10

\section{Introduction}

Heart failure (HF) is a leading cause of cardiovascular hospitalization and mortality. Mitral regurgitation (MR) and tricuspid regurgitation (TR) can both cause and exacerbate HF with increased morbidity and mortality (1). MR and TR can each be characterized by two distinct etiologies: primary degenerative valve disease, and secondary functional valve disease. Degenerative MR (DMR) is characterized by valvular or chordal degeneration with a core mechanism of systolic excessive leaflet movement defined by prolapse and/or flail affecting one or both leaflets and one or more scallops (2). Functional MR (FMR) is characterized by left ventricular (LV) remodeling due to ischemic or nonischemic myocardial disease $(3,4)$, annular dilatation, and leaflet malcoaptation. Tricuspid regurgitation is commonly encountered in the setting of pulmonary hypertension with associated right ventricular (RV) dilatation, tricuspid annular dilatation and tricuspid leaflet malcoaptation (5). The Cardioband valve reconstruction system (Edwards
Lifesciences, Irvine, CA, USA) is a catheter-delivered annulus-reducing technology that is most commonly applied to patients with MR or TR with the underlying pathophysiological mechanism of annular dilatation. Therefore, the initial Cardioband investigations focused on FMR and have naturally expanded into functional tricuspid regurgitation (FTR).

FMR affects a significant proportion of HF patients. Once MR becomes severe, it is associated with substantial 1-year mortality (6). FMR increases left atrial pressure, diminishes LV stroke volume, adds volume overload to a compromised left ventricle, and is associated with reduced survival. Guidelinedirected medical therapy (GDMT) may reverse cardiac remodeling, and is considered the initial standard of care for HF and associated FMR (7). However, HF complicated by FMR refractory to GDMT is associated with a poor prognosis and markedly diminished quality of life (3).

While approximately $60-70 \%$ of mitral valve surgeries in the United States are performed for the treatment of DMR (8), FMR accounts for a large proportion of patients with MR 


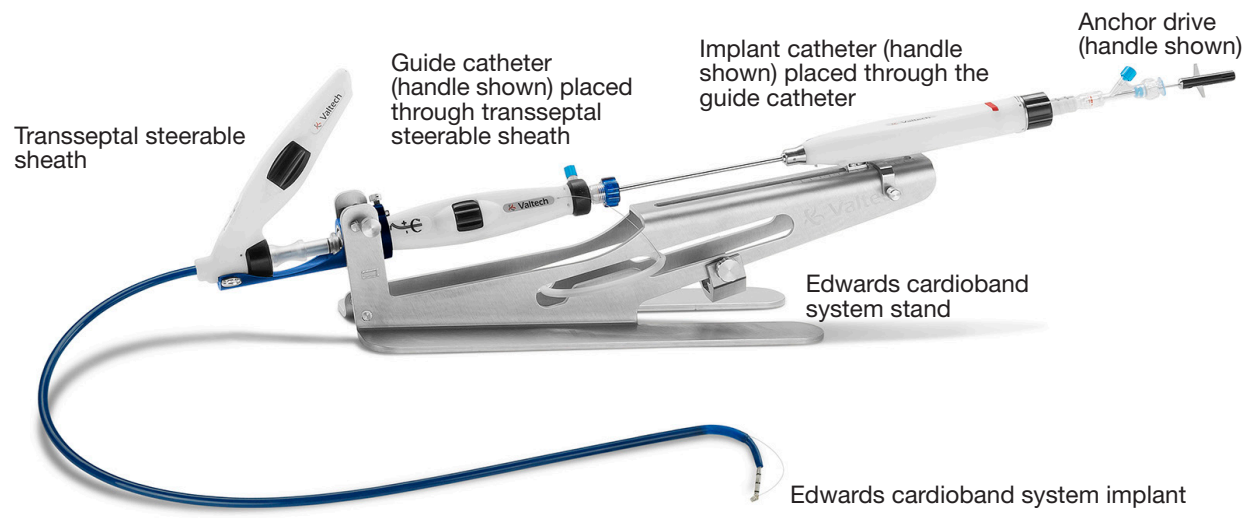

Figure 1 The mitral valve reconstruction Cardioband system.

(9). While surgical repair or replacement of the functionally regurgitant mitral valve is associated with improved quality of life, surgical therapy has not demonstrated a survival benefit, and has been shown in some patients to be associated with a precipitous decline in LV systolic function (10). The benefit of surgery in addition to GDMT has not been defined in a prospective randomized clinical trial. Surgical treatment of FMR is thus largely reserved for patients who require concomitant coronary artery bypass surgery or are considered low risk for cardiac surgery. Consequently, there is an unmet need for less invasive treatments of FMR with high quality prospective evidence supporting such interventions. Catheter-delivered devices including Cardioband seek to fill this void.

Tricuspid regurgitation and associated right heart failure are increasingly recognized as a major source of cardiovascular morbidity and mortality. However, the indications for and benefits of treating TR remain poorly defined. As tricuspid regurgitation is frequently the result of pulmonary hypertension with associated RV dilatation, many patients with indications for tricuspid repair have underlying co-morbidities and a higher surgical risk. In the United States, there are more than 1.6 million patients with moderate to severe tricuspid regurgitation (11), but less than 8,000 tricuspid surgical procedures are performed annually (12). Consequently, there remains an unmet clinical need for less invasive tricuspid repair options. Catheter-delivered devices like the Cardioband system hope to deliver a safe and efficacious tricuspid valve repair, and to define the indications and benefits of treatment.

Transcatheter edge-to-edge mitral leaflet repair is a safe and efficacious option for high-risk surgical patients with either DMR or FMR (13) and is increasingly studied for TR. However, transcatheter edge-to-edge repair does not reduce MR or TR as effectively as surgery. Edge-toedge repair reduces valve area, which may limit adoption in patients with certain mitral valve anatomy (e.g., those with a smaller mitral valve orifice). The edge-to-edge repair is being applied to patients with TR, but long-term data and efficacious application with a three-leaflet tricuspid valve has not been demonstrated.

The Cardioband system addresses the underlying FMR and FTR pathophysiological mechanism of annular dilatation with a minimally invasive, catheter delivered approach. The beating heart annulus is reduced and optimized under direct transesophageal echocardiography (TEE) visualization. This is a review of the Cardioband system and clinical experience to date, clinical benefits and future directions of this technology.

\section{The Cardioband mitral valve reconstruction system}

The Cardioband system includes the implant, delivery system, and reusable accessory components to facilitate the implantation procedure for the mitral valve (Figure 1). The Cardioband implant is anchored along the posterior annulus of the MV under beating heart condition. The Cardioband implant is made of a contraction wire and polyester fabric covering and is anchored into position by a series of stainless steel anchors. The Cardioband is available in six lengths to cover a wide range of annulus circumference sizes, comparable to surgical annuloplasty devices. After anchoring, the Cardioband implant is contracted under fluoroscopic and echocardiographic guidance to achieve a targeted anatomic annulus constraint and associated 


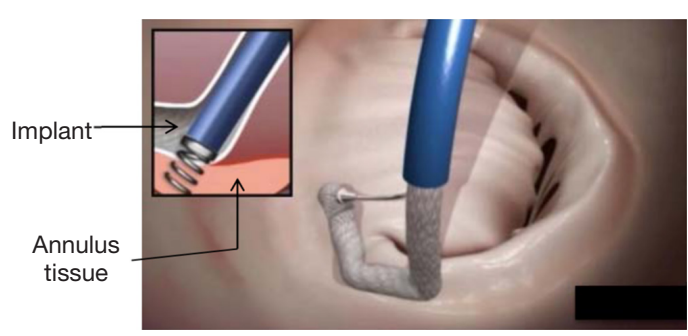

Figure 2 Anchor deployment through implant to the tissue.

optimization of valvular regurgitation.

The Cardioband delivery system includes the transseptal steerable sheath (TSS), the guide catheter (GC) and the implant catheter (IC).

\section{The Cardioband mitral annular reduction procedure}

The mitral valve is accessed through a percutaneous, transcatheter approach via the femoral vein and inferior vena cava. A transseptal puncture is performed in accordance with standard percutaneous techniques under TEE guidance, targeting the interatrial septum over the posterior medial mitral commissure. The TSS is advanced over a guide wire into the left atrium. The implant delivery system is advanced through the TSS into the left atrium and maneuvered to align the tip of the IC to the optimal position near the anterior lateral trigone under three-dimensional TEE (3D TEE) guidance. A series of anchors are advanced through the implant fabric and into the annular tissue in a stepwise fashion (Figure 2). Three anchors are deployed initially in the trigone near the anterior lateral commissure to ensure the Cardioband system implant is secured prior to further advancement of the implant along the commissure. The IC tip is maneuvered to each sequential target anchoring point along the posterior annulus using echocardiographic and fluoroscopic guidance. Two and three-dimensional TEE precisely guide each consecutive anchor through the implant to the annulus tissue, just adjacent to the mitral valve hinge point. When the last anchor is deployed in the trigone near the posterior medial commissure (12 to 17 anchors depending on implant length), the implant is disconnected from the IC.

After deployment of the Cardioband implant, the Size Adjustment Tool (SAT) is inserted through the TSS, over the SAT wire, to the adjustment mechanism of the implant. Rotating the adjustment knob reduces the implant length, which results in a controlled reduction of the implant and diminished MR as assessed by TEE under beating heart conditions. When the appropriate implant size has been achieved and MR optimized (Figure 3) the SAT and TSS are removed, leaving the implant deployed in place (Video: Cardioband, https://www.youtube.com/ watch?v=s0q5zH1SHw4).

\section{FMR patient selection}

Patients with FMR under consideration for Cardioband are screened with TEE and cardiac computed tomographic angiography (CTA). The TEE acoustic windows must allow for targeted transseptal puncture and visualize the annulus with sufficient resolution to define the hinge point between the annulus and the leaflet. The computed tomography (CT) analysis ensures that the annulus is of appropriate size, that the anchoring region of the mitral annulus is free of significant calcium, and that sufficient annular tissue is available for anchor placement without compromising the circumflex artery.

\section{Cardioband feasibility trial and early clinical experience}

The Cardioband system received CE Mark in September 2015 based on a European prospective multicenter study that established the safety and performance of the device. Safety was deemed to be acceptable, given the initial stage of the clinical experience and the high-risk profile of the population treated. There were no device or procedure related deaths. One patient with inadequate MR reduction underwent elective mitral surgery and died of surgical complications. One myocardial infarction was related to the interaction between an anchor and the circumflex artery. There were two major bleeding complications, including one tamponade.

Contraction of the implanted Cardioband reduced the annular septolateral dimension by $>30 \%$ from $3.7 \pm 0.5 \mathrm{~cm}$ at baseline to $2.5 \pm 0.4 \mathrm{~cm}$ after 1 month and to $2.4 \pm 0.4 \mathrm{~cm}$ after 6 months, respectively $(\mathrm{P}<0.001)$. At one month postoperatively, the percentage of patients with FMR $\geq 3$ was reduced from $77.4 \%$ to $10.7 \%(\mathrm{P}<0.001$, Figure 4$)$ and $13.6 \%(\mathrm{P}<0.001)$ at 6 months. The percentage of patients with New York Heart Association functional class III/IV HF decreased from $95.5 \%$ to $18.2 \%$ after 6 months $(\mathrm{P}<0.001)$. Exercise capacity as assessed by 6-minute walk test (6MWT) increased from $250 \pm 107$ to $332 \pm 118 \mathrm{~m}(\mathrm{P}<0.002)$, and quality of life, assessed by the Minnesota Living With Heart Failure Questionnaire (MLWHF improved from 38.2 \pm 21 to 

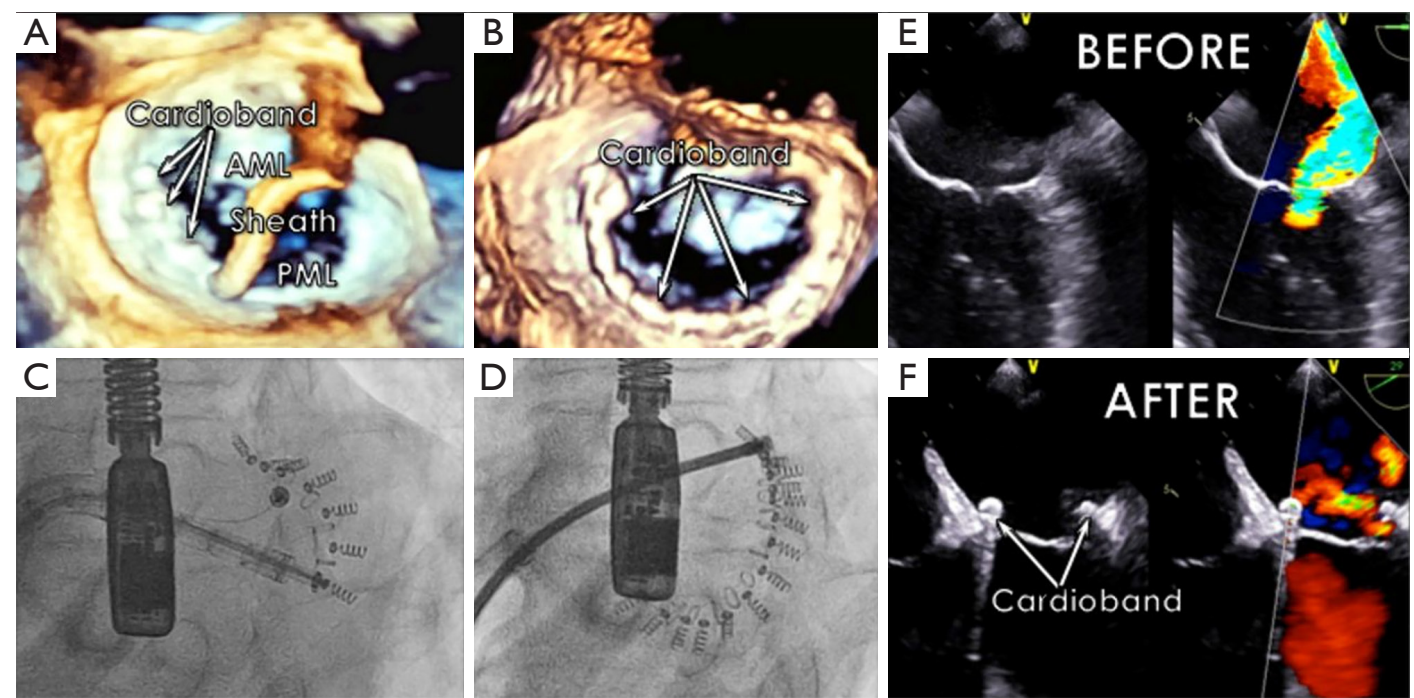

Figure 3 Corresponding views from 3D TOE and fluoroscopy with stepwise development of the device from the lateral commissure of the mitral valve to the $\mathrm{P} 2$ segment of the posterior mitral annulus $(\mathrm{A}, \mathrm{C})$; complete device development (B,D); severity of mitral regurgitation before the procedure and after annuloplasty (E,F). AML, anterior mitral valve leaflet; $\mathrm{MV}$, mitral valve; $\mathrm{P} 2$, posterior segment of the $\mathrm{MV}$; PML, posterior mitral valve leaflet; TOE, transesophageal echocardiography (14).

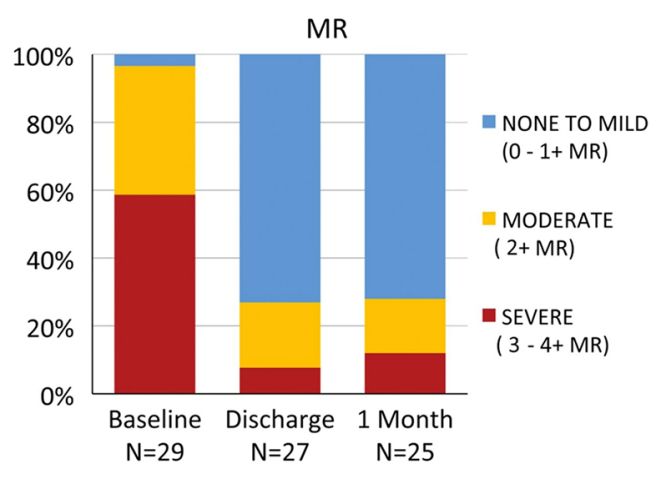

Figure 4 Mitral regurgitation reduction by transesophageal echocardiography at baseline $(\mathrm{N}=29)$ discharge $(\mathrm{N}=27)$ and 1-month follow-up (N=25). Twenty-seven patients were analyzed at discharge; two patients died prior to discharge and echo results were uncertain by core lab for two patients. Twenty-five of the 31 patients were analyzed at 1-month follow-up, two died prior to discharge, one withdrew prior to 1-month follow-up, the echo results were uncertain for two patients (4).

$18.1 \pm 10.9$ at 6 months $(\mathrm{P}<0.001)(14)$.

\section{The ACTIVE Trial}

The ACTIVE (Annular Reduction for Transcatheter
Treatment of Insufficient Mitral Valve) Trial is ongoing in the United States (ClinicalTrials.gov Identifier: NCT03016975) (15). The objective of this clinical trial is to evaluate the safety and efficacy of the Cardioband system for transcatheter mitral annular reduction in patients with clinically significant FMR (4). This is a prospective multicenter, randomized, controlled, and pivotal trial, which compares transcatheter mitral valve repair with the Cardioband system associated with GDMT compared to GDMT alone in patients with FMR. The primary endpoints are prevalence of $M R \leq 2+$ and a hierarchical composite of cardiovascular death, HF hospitalization, 6MWT and KCCQ at 1 year. Furthermore, all patients will be followed for 5 years to assess long-term outcomes. The study will randomize 375 patients in a 2:1 randomization scheme (250 devices vs. 125 controls).

The primary safety endpoint is the rate of device and procedure related major adverse events (MAEs) through 30 days post-procedure. The ACTIVE Trial inclusion criteria were selected to identify symptomatic HF patients with clinically significant FMR patients despite GDMT, likely to benefit from Cardioband MR reduction. Key inclusion criteria include:

(I) Age $>18$ years;

(II) FMR $\geq 3+$ as assessed by core lab echocardiography; 
(III) NYHA class II, III or ambulatory IV;

(IV) Symptomatic HF despite GDMT;

(V) Patient hospitalized due to HF during 12 months prior to submission or elevated brain natriuretic peptide (BNP) or NT pro BNP;

(VI) Patient is able and willing to give informed consent and follow protocol procedures, and comply with follow-up visit compliance;

(VII) Patient is deemed appropriate for Cardioband system by the heart team at the investigational site and the Central Screening Committee.

\section{The Cardioband tricuspid valve reconstruction system}

Tricuspid regurgitation may be due to degenerative valve disease, but is more often the result of increased RV pressure and often accompanies mitral or aortic valve disease. Tricuspid regurgitation has traditionally been considered less clinically significant than left side valvular heart disease. However, TR is associated with dyspnea, edema, hepatic congestion and dysfunction, RV failure, and excess mortality (16). While there is growing experience with surgical repair of the tricuspid valve, surgical tricuspid repair is associated with substantial morbidity and mortality (17).

As the Cardioband delivery system enables catheter movement in several dimensions, a dedicated system was developed to place the same implant in the right side for treatment of tricuspid regurgitation. In the European TRIREPAIR study interim analysis, the mean tricuspid valve annulus septolateral diameter was acutely reduced from $44.4 \pm 4.4$ to $37.0 \pm 4.5 \mathrm{~mm}$. Core lab echocardiographic assessment showed a $48 \%$ reduction in PISA EROA, a $27 \%$ reduction in vena contracta, and a $4 \%$ improvement in $\mathrm{LV}$ stroke volume from baseline to 6 months. The number of patients in NYHA class III/IV HF and with peripheral edema were significantly reduced at both 30 days and 6 months compared to baseline. Clinically significant improvements in exercise capacity and quality of life were observed at 30 days and sustained at 6 months. The $6 \mathrm{MWT}$ improved by $49 \mathrm{~m}$ and the KCCQ score by 24 points at 6 months (18). Similar efficacy has been published in case reports (19). The primary safety endpoint of major serious adverse events (MSAEs) were defined as any instances of death, myocardial infarction, cardiac tamponade, device-related cardiac surgery, and stroke within 30 days of the index procedure. Three subjects experienced 4 MSAEs ( 2 deaths, 2 cardiac tamponade and 1 stroke) within 30 days of the procedure.
The US Early Feasibility Study (EFS) of the Cardioband tricuspid system is a prospective, single arm, multi-center study to evaluate the safety and device functionality of the Cardioband tricuspid system (ClinicalTrials.gov Identifier: NCT03382457) (20). Symptomatic patients with chronic functional TR graded as moderate or greater despite medical therapy will be considered for enrollment. Patients with unsuitable anatomy, primary tricuspid valve disease, and/or previous tricuspid valve repair or replacement will be excluded.

\section{Discussion}

Both surgical and transcatheter mitral valve repair have been shown to benefit many FMR patients with improved hemodynamics, HF symptoms, and echocardiographic metrics including reverse $\mathrm{LV}$ remodeling with diminished $\mathrm{LV}$ end systolic index (LVESI) $(6,21,22)$. However, prospective, randomized evidence of clinical benefit is lacking. The results of several US and European MitraClip (Abbott, Abbott Park, IL, USA) FMR trials are anticipated in 2018 (COAPT and MITRA-FR) and 2019 (RESHAPE-HF-2) and may provide this evidence. However, enthusiasm for the MitraClip FMR trials must be tempered, as much has been learned in the course of these trials regarding patient selection and efficacy endpoints. Because transcatheter mitral valve repair is a procedure with associated periprocedural risks beyond those of medications, a conservative approach was taken for these early trials, limiting enrollment to those who failed to respond to coronary revascularization, aggressive GDMT, and, if indicated, biventricular pacing (9). Contemporary FMR device trials, including the ACTIVE Trial, are designed to engage the local heart team to identify and enroll symptomatic HF patients despite GDMT. Efficacy is assessed by aggregating several potential therapeutic benefits into a primary efficacy endpoint.

Furthermore, results from the MitraClip studies, which predominantly treat patients with $\mathrm{DMR}$, indicate that some patients can be left with residual MR, making it difficult to extrapolate these results to patients with FMR, and to other devices. The next-generation edge-to-edge MitraClip NT is now undergoing clinical investigation as is the novel PASCAL transcatheter mitral valve repair system (Edwards Lifesciences, Irvine, CA, USA) that utilizes individually adjustable clasps to place a spacer between the native mitral valve leaflets. With increasing experience, improved TEE guidance, and improved devices, the results of these mitral valve therapies will continue to improve. 
In the future, once devices have been approved, transcatheter mitral valve repair may not be considered in terms of a single device, but rather as a therapy, which may combine devices to target the mitral annulus, mitral leaflets, and/or chordae tendineae. Surgical mitral annular reduction is considered a foundational intervention for FMR as it not only reduces MR without causing mitral stenosis, but also decreases wall stress and constrains the $L V$ from further dilatation with favorable reverse remodeling. Surgical annuloplasty rings are commonly used with leaflet resection and are occasionally used in conjunction with Alfieri type edge-to-edge repair (23). The Cardioband system has been shown to replicate the benefits of annuloplasty and could be similarly anticipated to be a foundational procedure that compliment additional transcatheter interventions. As a first-line therapy, Cardioband has the advantage of allowing future transcatheter valve replacement, if indicated. It has already been combined in a few cases with transcatheter edge-to-edge repair and transapical chordal replacement (24). In the future, devices and procedures may be combined and tailored to each patient's unique anatomy.

There is debate regarding the absolute benefit of intervening on the functional regurgitant mitral valve in the setting of $\mathrm{HF}$, and also on whether repair or replacement is preferable. The NHLBI Cardiothoracic Surgical Trials Network (CTSN) demonstrated that when patients with severe FMR were randomized between restrictive mitral valve repair and chordal-sparing mitral valve replacement, there was no significant difference in the primary endpoint of LVESVI. However, patients randomized to repair were more likely to develop recurrent moderate or severe MR (58.8\% repair vs. $3.8 \%$ replacement) at 2 years, which translated into more HF related adverse events and cardiovascular hospitalizations. In the repair group, patients without recurrent moderate or severe MR had a greater degree of reverse remodeling than did patients who had such regurgitation (21). These studies seem to support replacement rather than repair for FMR. However, MV replacement has known limitations including a finite lifespan. Mitral repair when successful is typically preferred because the procedure is associated with superior safety, a better hemodynamic profile and indefinite durability $(25,26)$.

\section{Conclusions}

The Cardioband system provides a catheter-delivered valve repair that reduces mitral or tricuspid regurgitation through annular reduction. Sequential anchors secure the
Cardioband implant to the mitral or tricuspid annulus. The Cardioband is contracted under TEE guidance until valvular regurgitation is optimized. In its initial clinical experience, Cardioband was safely deployed in patients with clinically significant FMR yielding diminished septolateral dimensions, durable MR reduction, improved quality of life and exercise capacity (14). The early and evolving Cardioband experience in TR is similarly promising. The ongoing pivotal ACTIVE Trial is designed to demonstrate the superiority of Cardioband and GDMT compared to GDMT alone in patients with clinically significant FMR for a composite hierarchical endpoint of cardiovascular mortality, HF hospitalization, quality of life, and exercise capacity. The Cardioband system has the advantage of preserving the patient's native anatomy, therefore keeping future options open, and should be considered as a first-line therapy. Foreseeing a potential future with multiple device therapies, the Cardioband has been combined with edge-toedge repair and chordal replacement devices to maximize MR reduction. Additional studies are warranted to evaluate the potential of combined therapies to deliver optimal MR reduction with maximal clinical benefit.

\section{Acknowledgements}

None.

\section{Footnote}

Conflicts of Interest: Dr. VH Thourani and Dr. B Whisenant are the Principal Investigators in the ACTIVE Trial and have received honoraria from Edwards Lifesciences for consulting unrelated to the ACTIVE Trial and the Cardioband system. M Miller has no conflicts of interest to declare.

\section{References}

1. Nishimura RA, Otto CM, Bonow RO, et al. 2014 AHA/ ACC guideline for the management of patients with valvular heart disease: a report of the American College of Cardiology/American Heart Association Task Force on Practice Guidelines. J Am Coll Cardiol 2014;63:e57-185.

2. Antoine C, Mantovani F, Benfari G, et al. Pathophysiology of Degenerative Mitral Regurgitation: New

3-Dimensional Imaging Insights. Circ Cardiovasc Imaging 2018;11:e005971.

3. Sannino A, Smith RL 2nd, Schiattarrella GG, et al. 
Survival and cardiovascular outcomes of patients with secondary mitral regurgitation: a meta-analysis of 53 studies. JAMA Cardiology 2017;2:1130-9.

4. Maisano F, Taramasso M, Nickenig G, et al. Cardioband, a transcatheter surgical-like direct mitral valve annuloplasty system: early results of the feasibility trial. Eur Heart J 2016;37:817-25.

5. Medvedofsky D, Aronson D, Gomberg-Maitland M, et al. Tricuspid regurgitation progression and regression in pulmonary arterial hypertension: implications for right ventricular and tricuspid valve apparatus geometry and patients outcome. Eur Heart J Cardiovasc Imaging 2017;18:86-94.

6. Bishawi M, Milano C, Gaca J, Glower D. Durability of ischemic mitral repair in 167 patients treated with a rigid, undersized annuloplasty ring. Presented at the 96th annual American Association for Thoracic Surgery meeting. Baltimore, Maryland, 2016.

7. Benjamin MM, Smith RL, Grayburn PA. Ischemic and functional mitral regurgitation in heart failure: natural history and treatment. Curr Cardiol Rep 2014;16:517.

8. Mick SL, Keshavamurthy S, Gillinov AM. Mitral valve repair versus replacement. Ann Cardiothorac Surg 2015;4:230-7.

9. Deuschl F, Schofer N, Lubos E, et al. Critical evaluation of the MitraClip system in the management of mitral regurgitation. Vasc Health Risk Manag 2016;12:1-8.

10. Weir W, Bolling S, Whisenant B, et al. Ask the Experts: What Is the Role of Mitral Repair in Heart Failure? Cardiac Innovations Today. January/February, 2018.

11. Singh JP, Evans JC, Levy D, et al. Prevalence and clinical determinants of mitral, tricuspid, and aortic regurgitation (the Framingham Heart Study). Am J Cardiol 1999;83:897-902.

12. Stuge O, Liddicoat J. Emerging opportunities for cardiac surgeons within structural heart disease. J Thorac Cardiovasc Surg 2006;132:1258-61.

13. Schueler R, Nickenig G, May AE, et al. Predictors for short-term outcomes of patients undergoing transcatheter mitral valve interventions: analysis of 778 prospective patients from the German TRAMI registry focusing on baseline renal function. EuroIntervention 2016;12:508-14.

14. Nickenig G, Hammerstingl C, Schueler R, et al. Transcatheter Mitral Annuloplasty in Chronic Functional Mitral Regurgitation: 6-Month Results With the Cardioband Percutaneous Mitral Repair System. JACC Cardiovasc Interv 2016;9:2039-47.

15. Edwards Cardioband System ACTIVE Pivotal Clinical
Trial (ACTIVE). Available online: https://clinicaltrials. gov/ct $2 /$ show/NCT03016975 ? id=NCT03016975+OR+N CT02981953\&rank=1\&load=cart, 2018.

16. Arsalan M, Walther T, Smith RL, et al. Tricuspid regurgitation diagnosis and treatment. Eur Heart J 2017;38:634-8.

17. Zack CJ, Fender EA, Chandrashekar P, et al. National Trends and Outcomes in Isolated Tricuspid Valve Surgery. J Am Coll Cardiol 2017;70:2953-60.

18. Maisano F. Transcatheter tricuspid valve repair: up to sixmonths results from the multicentre trial. Presented at: EuroPCR 2018; May 22-25, 2018; Paris, France.

19. Schueler R, Hammerstingl C, Werner N, et al. Interventional Direct Annuloplasty for Functional Tricuspid Regurgitation. JACC Cardiovasc Interv 2017;10:415-6.

20. TRI-REPAIR: TrIcuspid Regurgitation RePAIr With CaRdioband Transcatheter System (TRI-REPAIR). 2016. Available online: https://clinicaltrials.gov/ct2/show/ NCT02981953

21. Goldstein D, Moskowitz AJ, Gelijns AC, et al. Two-Year Outcomes of Surgical Treatment of Severe Ischemic Mitral Regurgitation. N Engl J Med 2016;374:344-53.

22. Michler RE, Smith PK, Parides MK, et al. Two-Year Outcomes of Surgical Treatment of Moderate Ischemic Mitral Regurgitation. N Engl J Med 2016;374:1932-41.

23. De Bonis M, Lapenna E, La Canna G, et al. Mitral valve repair for functional mitral regurgitation in endstage dilated cardiomyopathy: role of the "edge-to-edge" technique. Circulation 2005;112:I402-8.

24. Latib A, Ancona MB, Ferri L, et al. Percutaneous Direct Annuloplasty With Cardioband to Treat Recurrent Mitral Regurgitation After MitraClip Implantation. JACC Cardiovasc Interv 2016;9:e191-2.

25. Matiasz R, Rigolin VH. 2017 Focused Update for Management of Patients With Valvular Heart Disease: Summary of New Recommendations. J Am Heart Assoc 2018;7:e007596.

26. Feldman T, Foster E, Glower DD, et al. Percutaneous repair or surgery for mitral regurgitation. $\mathrm{N}$ Engl J Med 2011;364:1395-406.

Cite this article as: Miller M, Thourani VH, Whisenant B. The Cardioband transcatheter annular reduction system. Ann Cardiothorac Surg 2018;7(6):741-747. doi: 10.21037/ acs.2018.10.10 